\title{
Síndrome de Brugada. Aspectos fisiopatológicos, clínicos y su asociación con enfermedades infecciosas
}

\author{
Juan Sebastián Rodríguez-Constaín', Nelson Adolfo López-Garzón², Carlos Alberto Navia-Amézquita³, \\ Diana Lorena Mora-Obando ${ }^{4}$, Rosa Amalia Dueñas-Cuellar ${ }^{5}$
}

\section{RESUMEN}

El síndrome de Brugada ( $\mathrm{SBr}$ ) es una enfermedad cardiaca no estructural que afecta los canales iónicos cardiacos, caracterizado por manifestaciones clínicas como arritmias, taquicardia, síncope y muerte súbita, entre otras. Su diagnóstico es netamente electrocardiográfico, con un patrón altamente sugestivo pero no patognomónico, por lo que existen diagnósticos diferenciales desde el punto de vista electrocardiográfico.

Existen tres patrones electrocardiográficos en los pacientes con $\mathrm{SBr}$, de los cuales el tipo I es el patrón más característico. Actualmente, múltiples genes se han relacionado con la presentación de este síndrome, entre los cuales se destaca el gen SCN5A, el más descrito en la literatura. Se conoce que este síndrome es más frecuente en el género masculino; sin embargo, no existen estudios epidemiológicos en Latinoamérica que lo confirmen. Pese a que la investigación alrededor de los mecanismos causales del síndrome ha avanzado, existen varias cuestiones sin resolver, como su desenmascaramiento por los signos que producen algunas enfermedades infecciosas causadas principalmente por virus. Por lo tanto, dada la relevancia clínica del tema para el médico general y para el especialista, el objetivo de esta revisión es describir no solo aspectos fisiopatológicos y clínicos de la enfermedad, sino también resaltar casos de pacientes con enfermedades infecciosas quienes posteriormente han sido diagnosticados con el síndrome de Brugada.

1 Estudiante, Programa de Medicina, Facultad de Ciencias de la Salud, Universidad del Cauca, Colombia.

2 Docente, Departamento de Medicina Interna, Facultad de Ciencias de la Salud, Universidad del Cauca, Colombia.

3 Docente, Departamento de Ciencias Fisiológicas, Facultad de Ciencias de la Salud, Universidad del Cauca, Colombia.

4 Estudiante de doctorado, Laboratorio de Venómica Evolutiva y Traslacional, Instituto de Biomedicina de Valencia, España.

5 Docente, Departamento de Patología, Facultad de Ciencias de la Salud, Universidad del Cauca, Colombia.

Correspondencia: Rosa Amalia Dueñas-Cuellar; raduenasc@unicauca.edu.co

Recibido: mayo 21 de 2018

Aceptado: septiembre 11 de 2018

Cómo citar: Rodríguez-Constaín JS, López-Garzón NA, Navia-Amézquita CA, Mora-Obando DL, Dueñas-Cuellar RA. Síndrome de Brugada. Aspectos fisiopatológicos, clínicos y su asociación con enfermedades infecciosas. latreia. 2019 Jul-Sep;32(3):217-231. D0I 10.17533/udea.iatreia.19. 


\section{PALABRAS CLAVE}

Electrocardiografía; Enfermedades Infecciosas; Síndrome de Brugada

\section{SUMMARY}

Brugada syndrome. Physiopathological, clinical aspects and association with infectious diseases

Brugada syndrome (BrS) is a non-structural cardiac disease that affects cardiac ion channels; it is characterized by clinical manifestations such as arrhythmias, tachycardia, syncope and sudden death, among others. Its diagnosis is mainly electrocardiographic, with a highly suggestive but not pathognomonic pattern, thus, there could be differential diagnoses from the electrocardiographic point of view.

There are three electrocardiographic patterns in patients with BrS, of which type I is the most characteristic pattern. Currently, multiple genes have be linked to the presentation of this syndrome, among which the SCN5A gene is the most described in the literature. It is know that this syndrome is more frequent in males; however, there are not epidemiological studies in Latin America that confirm it. Although research around the causal mechanisms of the syndrome has advanced, there are several unresolved issues, for example, its unmasking by the sions produced for some infectious diseases caused mainly by viruses. Therefore, given the clinical relevance of the topic, for the medical general practitioner and the specialist, the objective of this review is to describe not only the physiopathological and clinical aspects of the disease, but also highlight cases of patients with infectious diseases, who subsequently have been diagnosed with Brugada syndrome.

\section{KEY WORDS}

Brugada Syndrome; Electrocardiography; Infectious Diseases

\section{INTRODUCCIÓN}

El Síndrome de Brugada (SBr) fue descrito por primera vez en 1992 por Pedro y Josep Brugada (1). Es una enfermedad cardiaca no estructural, causante del 4 al $12 \%$ de todas las muertes súbitas en personas que generalmente no presentan ninguna sintomatología; entre el 20 y el $50 \%$ de estas muertes ocurren en pacientes sin ninguna enfermedad cardíaca estructural demostrable y como consecuencia de una arritmia (2-4).

Las bases genéticas de la enfermedad han demostrado que su origen es de carácter autosómico dominante y se han aislado aproximadamente 20 genes asociados con más de 250 mutaciones $(5,6)$. Sin embargo, existen diagnósticos de novo en los que el cribado de la enfermedad en familiares directos no encuentra antecedentes (7). Los principales síntomas incluyen: taquicardia, síncope, arritmias y muerte súbita como desenlace final (8).

Algunas investigaciones sugieren que los pacientes asintomáticos presentan un mayor riesgo de sufrir muerte súbita debido a la falta de un diagnóstico oportuno (9), otras manifiestan que la presencia de síntomas es un factor de riesgo del síndrome $(10,11)$ y otras, que la evolución es similar tanto en pacientes sintomáticos como en asintomáticos (12). Por lo tanto, se debe catalogar como paciente de alto riesgo a todo aquel que presente patrones electrocardiográficos (ECG) característicos del síndrome (13). Sin embargo, cabe resaltar que el estudio electrofisiológico está altamente cuestionado como método de estratificación de riesgo en el síndrome de Brugada $(14,15)$.

Se han descrito tres patrones electrocardiográficos en el SBr, de los cuales el tipo I es el patrón característico del síndrome (16). Aunque estos patrones son la base para el diagnóstico deI $\mathrm{SBr}$, algunos pacientes presentan una forma oculta o intermitente, la cual necesita de otros métodos para su diagnóstico (17).

Se estima que la prevalencia del SBr es de 1 por cada 2.000 individuos en el mundo. Sin embargo, existen diferencias en estas cifras dependiendo de la ubicación geográfica (18); por ejemplo, en países occidentales como España, Italia, Bélgica, Países Bajos, Grecia, Alemania, Austria, Suiza, Polonia, Ucrania y Francia, la prevalencia aproximada es de 1 a 5 casos por cada 10.000 habitantes $(19,20)$, mientras que en los países orientales 1 de cada 2.500 personas presentan el síndrome (21). Algunos de estos casos se han reportado en ciudades como Japón, Tailandia, China, India, Laos, Vietnam, Singapur y Camboya (22). 
En el continente americano no se han realizado estudios epidemiológicos en el tema, por lo que se desconoce la cifra exacta de casos, pese a esto, algunos casos han sido reportados en países como Estados Unidos, Canadá, Brasil, Argentina y Uruguay (23-27). En Colombia se han reportado casos en Bogotá (28), Cali (29) y Medellín (18), no obstante, el número de casos para el país podría estar subestimado. En la Ciudad de Popayán, por ejemplo, aunque existen observaciones clínicas que sugieren su presencia, los casos no han sido publicados.

Factores como el género, pueden influir en la presentación clínica de la enfermedad $(30,31)$. Estudios epidemiológicos sugieren que existe una mayor prevalencia en el sexo masculino que en el femenino en una relación de 8:1 (32), probablemente por la acción reguladora de las hormonales sexuales, que permiten una mayor expresión de genes sensibles a hormonas esteroideas, en especial la testosterona (33), la cual favorece una mayor expresión de canales de sodio a nivel cardiaco $(34,35)$.

En este sentido, se ha encontrado que en alounos pacientes con síndrome de Brugada, los altos niveles de testosterona se asocian con la presencia del fenotipo característico del $\mathrm{SBr}$ y explican el predominio del síndrome en el sexo masculino (36). Además, dichos pacientes se caracterizan por tener un menor porcentaje de grasa visceral, secundaria a este influjo hormonal por mayores niveles de testosterona $(37,38)$.

Los hombres con síndrome de Brugada presentan un perfil clínico de mayor riesgo que las mujeres y tienen un peor pronóstico $(39,40)$. Otros estudios han descrito que en mujeres se presenta con menor frecuencia el patrón ECG tipo I, siono característico de la enfermedad (41). Pese a lo anterior, la información sobre las diferencias existentes en cuanto al género en el SBr es escasa y no es totalmente clara (42).

En pacientes con algún tipo de infección, ya sea viral, parasitaria o bacteriana, se han encontrado patrones electrocardiográficos característicos del $\mathrm{SBr}$ como resultado de la elevación de la temperatura normal, elevación que puede acelerar la inactivación de los canales de sodio, reproduciendo el fenómeno electrocardiográfico de tipo I (43). En la literatura se reportan múltiples casos de pacientes en los cuales, por síntomas como la fiebre causada por enfermedades infecciosas, se logra desenmascarar un SBr oculto (44-48).

\section{FISIOPATOLOGÍA Y PATOGÉNESIS DEL SÍNDROME DE BRUGADA}

El corazón es un órgano muscular cuya función principal es proveer sangre a todos los tejidos del cuerpo humano. Electrofisiológicamente se caracteriza por presentar un potencial de acción de aproximadamente $-85 \mathrm{mvol}$ en reposo, y logra alcanzar un potencial de membrana aproximado de $20 \mathrm{~m} v 0 \mathrm{l}$ (despolarización) en cada latido.

La despolarización cardiaca se ha dividido en 5 fases $(0-4)$, cada una de las cuales está determinada por la entrada y salida de los iones $\mathrm{K}^{+}, \mathrm{Na}^{+}, \mathrm{Ca}^{+2}$ (Figura 1. A) (49). La fase 0 se caracteriza por la entrada de $\mathrm{Na}^{+}$al espacio intracelular a través de canales dependientes de voltaje, $y$ al mismo tiempo ocurre la apertura de canales lentos de $\mathrm{Ca}^{+2}$, esto hace que la célula cardiaca se despolarice y su potencial de membrana aumente hasta alcanzar la espiga o punta (20 mvol), momento en el cual se cierran todos los canales de $\mathrm{Na}^{+}$. Después de la inactivación de los canales de $\mathrm{Na}^{+}$ocurre la salida de $\mathrm{K}^{+}$y la entrada de $\mathrm{Cl}^{-}$, lo que define la fase $1 \mathrm{del}$ potencial del miocardio.

Durante esta fase, de corta duración, ocurre una rápida repolarización en la que el potencial de membrana retorna a 0 mvol. La fase 2 , también conocida como fase de meseta cardiaca, tarda entre 0,2 y 0,3 segundos y da lugar a la entrada de $\mathrm{Ca}^{+2}$ y $\mathrm{Cl}$.

Durante la fase 3 se cierran los canales lentos de $\mathrm{Na}^{+}$y $\mathrm{Ca}^{+2}$ y aumenta la salida de $\mathrm{K}^{+}$, como resultado, ocurre una repolarización rápida que lleva a la célula nuevamente a su potencial negativo (-85 mvol). Finalmente se presenta la fase 4 , la cual permite el equilibrio químico de los iones a nivel intra y extra celular, esta restitución electrolítica se logra por acción de la bomba Na-K-ATPasa, la cual expulsa el exceso de $\mathrm{Na}^{+}$que se encuentra a nivel intracelular e ingresa $\mathrm{K}^{+}$por transporte activo, conservando el potencial negativo de la célula, asimismo el intercambiador $\mathrm{Na}^{+} / \mathrm{Ca}^{+2}$ permite la salida de un ión $\mathrm{Ca}^{+2}$ por la entrada de 3 iones de $\mathrm{Na}^{+}$(Figura 1. A y D) (50-52).

En el SBr, el potencial de acción normal muestra alteraciones y hasta el momento se han descrito dos hipótesis que explican su implicación en la patogénesis. La primera, conocida como deterioro de la repolarización, explica que el desbalance de cargas positivas por alteración de las corrientes iónicas de la fase 1 del potencial conduce a una pérdida de la meseta, acortándola en un 


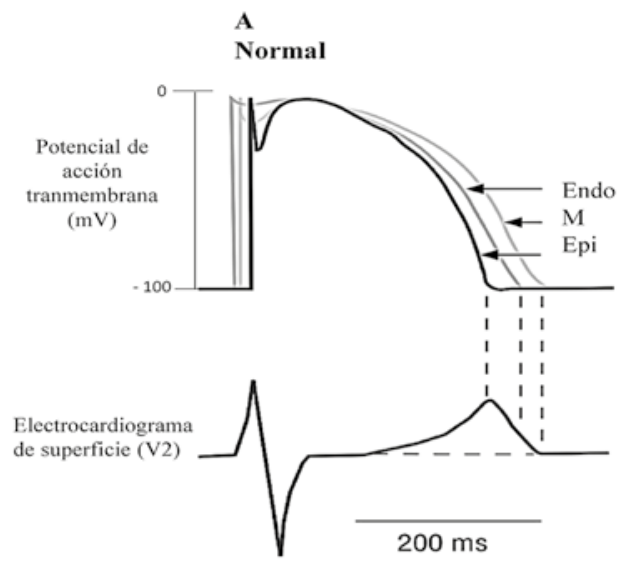

D

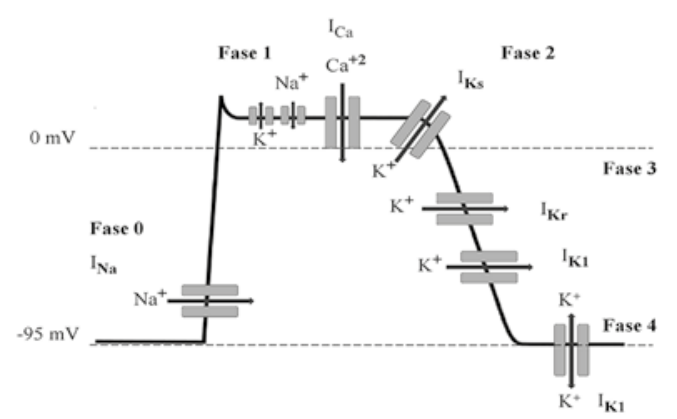

B

Patrón tipo I
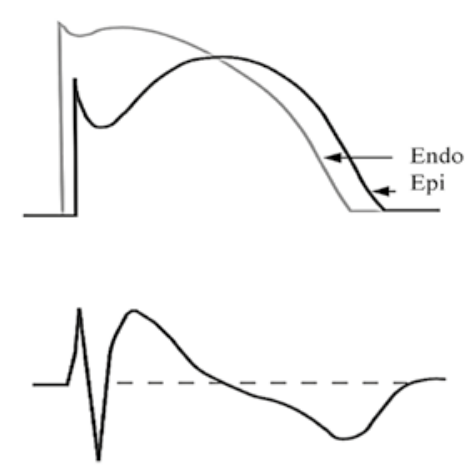

E
C
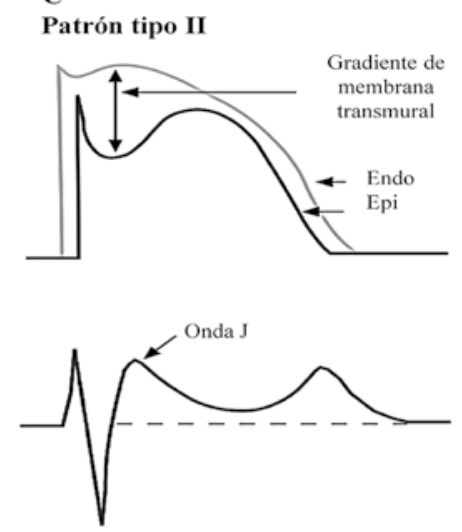

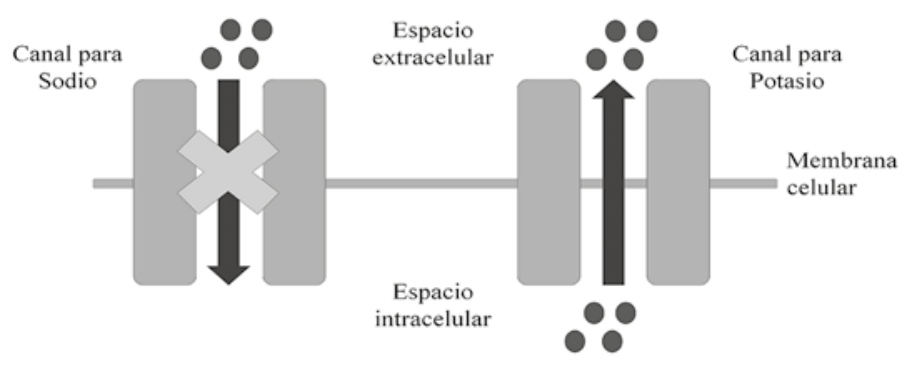

Figura 1. Potenciales de acción asociados a los patrones electrocardiográficos del síndrome de Brugada. A. Potencial de acción normal del corazón. B. Potencial de acción asociado al patrón tipo I. C. Potencial de acción asociado al patrón tipo II. D. Acción normal de los canales durante el potencial de acción. E. Bloqueo del canal de sodio que originan los patrones electrocardiográficos del síndrome de Brugada. Fuente: adaptada de Brugada et al. (2009)

40 a $70 \%$ en el epicardio (Figura 1). B) $(53,54)$. Esto se puede desencadenar como resultado de la disminución de las corrientes de entrada de $\mathrm{Ca}^{+2}$ o de $\mathrm{Na}^{+} \mathrm{O}$, por un aumento de las corrientes de salida transitorias de $\mathrm{K}^{+}$ entre el epicardio derecho y el endocardio, lo que ocasiona una dispersión transmural de la repolarización y la refractariedad, dando lugar al patrón ECG típico (tipo I), donde se presenta la elevación del ST $(21,55)$ observada en derivaciones precordiales derechas (56).

La segunda hipótesis se denomina teoría de la despolarización, esta se presenta secundaria a un retraso de la conducción en la región anterior epicárdica del tracto de salida del ventrículo derecho. Esto se explica por la existencia de alounas zonas con potenciales anormales con un bajo voltaje y duración prolongada, algo que no ocurre a nivel del endocardio anterior del mismo tracto de salida, ni en otras áreas de los ventrículos derecho e izquierdo (Figura 1. C) (57). Las arritmias y la muerte súbita que caracterizan al $\mathrm{SBr}$ se deben al desarrollo de un mecanismo de reentrada en la fase 2, como resultado de la heterogeneidad y dispersión de la repolarización, tanto a nivel transmural como epicárdico, con una mayor vulnerabilidad en la aparición de complejos ventriculares prematuros (58). El corazón tiene un actividad eléctrica normal, conocida como ciclo cardiaco, en el cual se despolarizan todas las células del corazón enviándolas a un estado refractario y así evitar una nueva excitación; sin embargo, si un grupo de fibras no se activan oportunamente en el ciclo, ya sea por el retraso o la activación precoz, pueden activar zonas previamente despolarizadas que se recuperaron de la activación inicial, por lo que se genera un segundo potencial, el cual producirá un 
fenómeno de reentrada y volverá a despolarizar ciertas células del corazón que ya han sido repolarizadas por el primer potencial (59). Todos estos fenómenos se pueden demostrar con la administración de flecainida $(60,61)$, un fármaco antiarrítmico que, junto con la ajmalina son bloqueadores del canal de sodio y, por lo tanto, son medicamentos esenciales para el diagnóstico deI SBr (62). EI test se considera positivo si durante la perfusión del fármaco en el paciente, el ECG ya sea tipo II o III, se convierte en ECG diagnóstico de tipo I, caracterizado por un ascenso del segmento ST $\geq$ $2 \mathrm{~mm}$ seguido de T negativa en más de una derivación precordial derecha $(\nabla 1-\nabla 3)(63,64)$.

Factores externos al corazón también pueden influir en la aparición de arritmias, por ejemplo el tono autonómico (65). Algunos autores describen un aumento súbito de la actividad vagal inmediatamente antes de la aparición de fibrilación ventricular, lo cual puede confirmar que una arritmia puede darse por diversos aspectos. Se ha informado de la existencia de una pérdida del balance entre el simpático y el parasimpático en el SBr. Cuando ocurre una reducción en la actividad simpática con predominio del tono parasimpático aumenta el riesgo de arritmias, esta podría ser la razón por la cual la incidencia de arritmias y muerte súbita se presentan en reposo o durante el sueño (donde hay un aumento del parasimpático), cabe resaltar que este fenómeno ocurre en sujetos con $\mathrm{SBr}$ y no en otras circunstancias patológicas en las cuales, por el contrario, el aumento del tono simpático es el generador de arritmias $(66,67)$.

\section{BASES GENÉTICAS}

El SBr tiene un patrón de transmisión autosómico dominante con penetrancia incompleta (68). Se conoce que el $25 \%$ de los pacientes presentan alguna mutación en el gen (3p21) (5). Este codifica la subunidad $\alpha$ del canal de sodio Nav1.5, que determina la fase 0 del potencial de acción cardíaco (69). Las mutaciones en este gen generan la pérdida de la función del canal de sodio reduciendo la corriente entrante del ion, lo que ocasiona las manifestaciones clínicas y el ECG característico del $\mathrm{SBr}$ (70). La subunidad $\alpha$ de este canal de sodio a su vez interactúa con la subunidad $\beta 1$, anquirina G, caveolina 3 y sintrofina; por lo tanto, las mutaciones en los genes que codifican para estas proteínas también se han asociado al síndrome (71).
Se han encontrado otras mutaciones relacionadas al $\mathrm{SBr}$ (72), por ejemplo la mutación en el gen SCN10A, que codifica para el canal de sodio Nav1.8, conduce a una pérdida de su función y disminuye la corriente de $\mathrm{Na}^{+}$por modulación de la actividad del canal Nav1.5 codificado por SCN5A, alteraciones que prolongan los intervalos PRI y QRS $(73,74)$. Otra mutación relacionada con el $\mathrm{SBr}$ es la que ocurre en el gen similar al glicerol-3-fosfato dehidrogenasa-1 (GPD1L), que actúa como modulador de los canales iónicos, aunque el mecanismo exacto es desconocido (75). Por otro lado, la mutación en el gen SCN1B42 evita la apertura del canal $\mathrm{Na}^{+}$y mutaciones en el gen SCN3B43 causan reducción de la corriente de $\mathrm{Na}^{+}$por interferencia en el tráfico normal del canal hacia la membrana celular (59). Recientemente, se ha demostrado que las mutaciones dominantes negativas en el gen RANGRF, el cual codifica para la proteína MOG1, perjudican la regulación de la expresión y el tráfico del canal de sodio hacia la membrana, conduciendo a la reducción de las corrientes de sodio y por tanto a las manifestaciones del síndrome (76).

También se han aislado mutaciones en los genes que codifican para las subunidades $\alpha-1$ (CACNA1C), $\beta$-(CACNB2b) y $\delta 1$ (CACNA2D1) del canal de $\mathrm{Ca}^{+2}$ cardiaco tipo L, causando una disminución de la corriente de entrada de $\mathrm{Ca}^{+2}$. Esta condición está relacionada con el 10-15\% de los casos de SBr y del síndrome de QT corto (77-80). Las mutaciones en los genes KCNE347 y KCNE5 causan el aumento de la corriente transitoria de salida de $\mathrm{K}^{+}$. Por último, las mutaciones en KCNJ8 bloquean el canal de potasio evitando la salida del ion $y$ dan como resultado la pérdida de la meseta cardiaca $(81,82)$.

\section{MANIFESTACIONES CLÍNICAS Y DIAGNÓSTICO}

Los pacientes con $\mathrm{SBr}$ no tienen ningún siogno de cardiopatía estructural evidente, pero presentan arritmias ventriculares malignas, síncope o muerte súbita cardíaca, que por lo general se suelen dar en el reposo o en el sueño nocturno (83). Además, pueden presentar episodios de taquicardia ventricular polimórfica (TVP) que terminan en fibrilación ventricular (FV). A la aparición de tres o más episodios de taquicardia ventricular o fibrilación ventricular separada, que requieren de terapia con desfibrilador automático 
implantable (DAI), en un período de $24 \mathrm{~h}$, se le conoce como tormenta eléctrica, fenómeno raro pero potencialmente fatal (84-86). Estos eventos arrítmicos ventriculares se observan con mayor frecuencia en la cuarta década de la vida, aunque se han descrito casos entre los 19 y 56 años (48, 87-89), así como en la población pediátrica (90).

Las manifestaciones clínicas del síndrome incluyen historial de síncope, respiraciones agónicas nocturnas durante el sueño, historia familiar de muerte súbita antes de los 45 años, fibrilación ventricular documentada, taquicardia ventricular polimórfica y la presencia del patrón electrocardiográfico tipo I en algún miembro de la familia (82); estas manifestaciones son útiles para direccionar la intervención terapéutica del paciente.

Actualmente el diagnóstico deI SBr se basa en criterios electrocardiográficos. Según el consenso vigente sobre diagnóstico y manejo de pacientes con síndromes de arritmias heredadas primarias de la Heart Rhythm Society y la European Heart Rhythm Association, se han descrito tres patrones ECG distintos para el SBr, los cuales se caracterizan por la presencia de una morfología semejante a un bloqueo de rama derecha, con la diferencia de que se encuentra una elevación del segmento ST en las derivaciones precordiales deI ventrículo derecho $(\nabla 1, \nabla 2)$, con los electrodos localizados en el 2do, 3ro o 4to espacio intercostal $(91,92)$.

El patrón de tipo I se caracteriza por una elevación convexa y descendente del segmento $\mathrm{ST} \geq 2 \mathrm{~mm}$ en más de una derivación precordial derecha $(\nabla 1-\nabla 2)$, con ondas T negativas, elevación que ocurre espontáneamente o después de administrar drogas antiarrítmicas de clase I por vía intravenosa. El patrón de tipo II se caracteriza por la elevación del segmento $\mathrm{ST} \geq 2$ $\mathrm{mm}$ en precordiales derechas seguida de ondas $\mathrm{T}$ positivas o isobifásicas (ECG aspecto de silla de montar) y, el patrón de tipo III se define como cualquiera de los dos anteriores pero con elevación del seomento ST $\leq 1 \mathrm{~mm}$ (Figura 2) (21, 93-95), dado que este patrón muestra una elevación deI ST no significativa, no es completamente sugestivo de esta canalopatía (7).
A

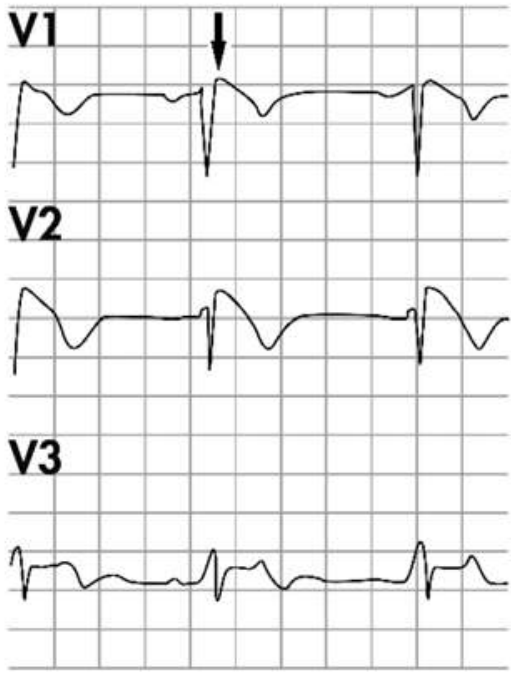

B
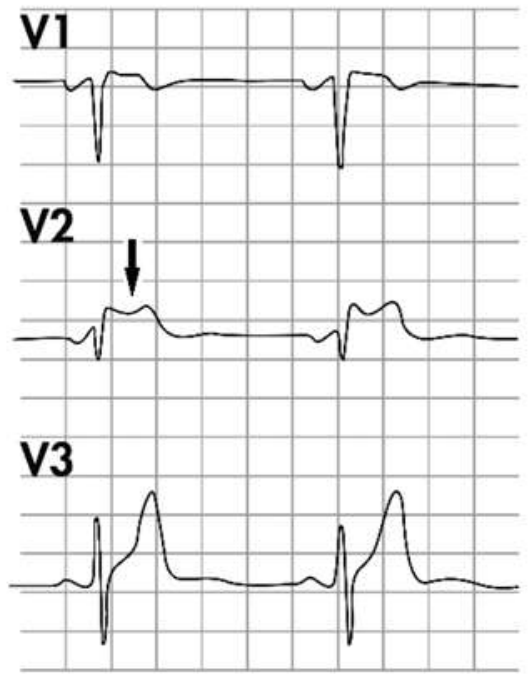

C
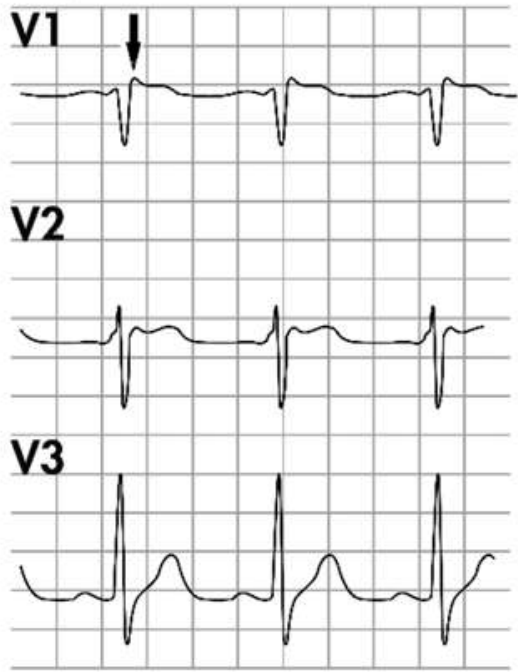

Figura 2. Patrones electrocardiográficos del síndrome de Brugada. A. Patron electrocardiografico tipo I, la flecha muestra la elevacion convexa y descendente del segmento $S T \geq 2 \mathrm{~mm}$ ondas T negativas. B. Patron electrocardiografico tipo II, elevacion del segmento $S T \geq 2 \mathrm{~mm}$ T positivas 0 isobifasicas, silla de montar (indicado con una flecha). C. Patrón electrocardiografico tipo III, la flecha indica la elevacion del segmento ST es $\leq 1 \mathrm{~mm}$. Fuente: adaptada de Brugada et al. (2009). 
Los dos últimos patrones son diagnosticados mediante test farmacológico con drogas antiarrítmicas clase 1 que inducen la morfología de un ECG tipo I $(62,63)$. para confirmar el SBr se debe realizar un ECG y evaluar si este corresponde al patrón electrocardiográfico tipo I, el cual es característico del síndrome. Se sugiere que todo paciente con patrón tipo I asintomático debe someterse a un interrogatorio exhaustivo, examen físico y registro de un ECG convencional y con derivaciones modificadas; también se recomienda estudiar a todos los familiares consanguíneos (96). En caso de que se presenten los patrones tipo II o III, se requiere la confirmación del diagnóstico mediante la realización del test de ajmalina, procainamida o flecainida (97).

Es importante resaltar que algunas enfermedades pueden presentar registros electrocardiográficos similares al SBr, por ejemplo el infarto agudo de miocardio, la miocarditis aguda, el infarto del ventrículo derecho, el trombo embolismo pulmonar agudo, la hiperpotasemia, la displasia arritmogénica del ventrículo derecho, los bloqueos de la rama derecha e izquierda, la hipertrofia ventricular izquierda y el aneurisma disecante de la aorta, por lo que se dificulta el diagnóstico diferencial (98).

Algunas condiciones como los episodios febriles pueden desenmascarar formas silentes del $\mathrm{SBr}$ y/o conferir un riesgo incrementado (transitorio) de arritmias ventriculares (99), especialmente en la población pediátrica (100), donde la identificación del SBr es poco habitual y la mayoría de los casos reportados se desenmascaran después de episodios febriles (101).

\section{DESENMASCARAMIENTO DEL SÍNDROME DE BRUGADA POR ENFERMEDADES INFECCIOSAS}

Varios casos de patrones electrocardiográficos de $\mathrm{SBr}$ han sido reportados en pacientes con algún tipo de infección, ya sea viral, parasitaria o bacteriana. Estas infecciones elevan la temperatura normal hasta llegar a la fiebre (temperatura matutina $>37,2^{\circ} \mathrm{C}$ o una temperatura vespertina $\left.>37,7^{\circ} \mathrm{C}\right)(46,102)$. Esta elevación de la temperatura puede acelerar la inactivación de los canales de sodio con una recuperación más lenta y ocasionar aparición de la supra desnivelación del segmento ST en las precordiales derechas (43).
A continuación, se describen algunos casos de pacientes con enfermedades infecciosas a quienes se les diagnosticó el síndrome luego de sufrir episodios de fiebre durante los cuadros clínicos de la enfermedad. Se ha reportado el caso de una paciente mujer de 51 años de Badajoz, España, con antecedentes personales de diabetes mellitus tipo II, fumadora de 3 cigarrillos al día y artrosis, que ingresa en el contexto de fiebre y dolor abdominal de 24 horas de evolución, localizado en la fosa ilíaca izquierda, acompañada de náuseas y vómitos alimentarios. En el examen físico se resalta que fue positivo la puño percusión, en el electrocardiograma presenta un patrón de Brugada tipo II durante los episodios febriles de una pielonefritis aguda por Enterobacter aerogenes aislada en hemocultivos y uroanálisis, cabe resaltar que el ECG se normaliza en los periodos afebriles (103).

En infecciones virales, donde se desarrolla un síndrome febril puede ocurrir una elevación del segmento ST con la aparición de algún patrón ECG de SBr, el más común en estos casos es el patrón tipo I, donde la disminución de la fiebre hace desaparecer el patrón (104). Los siguientes casos describen la anterior situación, el primero es un hombre de 49 años, quien fue admitido para investigación de síncopes recurrentes, episodios que habían comenzado tras la infección por el virus H1N1 (pandemia del año 2009) y que continuaron apareciendo durante cuadros febriles de una neumonía pos influenza.

Un electrocardiograma de 12 derivaciones (ECG) (Figura 1), grabado mientras el paciente estaba febril (temperatura $38,3^{\circ} \mathrm{C}$ ), mostró una elevación del segmento ST en derivaciones $\nabla 1-\nabla 3$, consistente con un patrón de tipo I. Tiempo después de que la fiebre se resolvió, el patrón electrocardiográfico desapareció (105). El segundo, es el caso de un hombre de 58 años de edad que se presentó en el hospital con una úlcera en la garganta, tos y fiebre de $38,5^{\circ} \mathrm{C}$; el ECG mostró una elevación del segmento ST en las derivaciones $\nabla 1$ a $\nabla 3$ respecto del electrocardiograma inicial, mientras que las enzimas cardíacas, la radiografía de tórax, la ecocardiografía bidimensional y la arteriografía coronaria fueron normales. Todos estos síntomas se atribuyeron a una enfermedad viral, la elevación del segmento ST se resolvió con la disminución de la fiebre (106). Adicionalmente, se reportan algunos casos de presentación del patrón tipo I en pacientes con malaria, y la desaparición del ECG patológico en el momento de la mejoría del paciente $(107,108)$. 
Al tener en cuenta lo anterior, es importante considerar como diagnóstico diferencial el $\mathrm{SBr}$ en pacientes que cursen un estado febril, presenten dolor precordial u otras características de infarto, acompañados de hallazgos electrocardiográficos sugestivos de síndrome de Brugada (109), ya que también se han reportado casos de patrón tipo II en el electrocardiograma por pericarditis. Por ejemplo, en un hombre de 27 años, de Perugia, Italia, el cual acudió al médico por fatiga e incomodidad en el pecho; los hallazgos del laboratorio mostraron un aumento del recuento de leucocitos y la proteína C-reactiva. El paciente ingresó con un diagnóstico de pericarditis, sin embargo, en el electrocardiograma se logró evidenciar una elevación del segmento ST de tipo "silla de montar" en la derivación $\nabla 2$, reconocida como patrón de Brugada tipo II. Unos días después del inicio de la terapia antiinflamatoria el ECG se normalizó (110). Se debe tener en cuenta que presentar un patrón de SBr cuando la temperatura está elevada aumenta el riesgo de presentar eventos arrítmicos y padecer un desenlace fatal por muerte súbita (111).

\section{TRATAMIENTO}

Cuando se sospecha de este síndrome en el paciente se recomienda evitar algunos factores de riesgo que incrementan la elevación del segmento ST, tales como los fármacos antiarrítmicos (procainamida, propafenona) (112), psicotrópicos (amitriptilina, litio) y anestésicos/analgésicos (procaina y propofol), entre otros (cocaína, alcohol) (113).

Actualmente, el SBr es tratado con ciertos fármacos o con el uso de un desfibrilador automático implantable (DAI) en pacientes sintomáticos (Tabla 1); sin embargo, en pacientes asintomáticos es muy difícil decidir una conducta terapéutica (114-116). Los fármacos disponibles son la quinidina, que ha probado ser útil en el tratamiento de pacientes que desarrollan tormentas eléctricas, así como el isoproterenol, la disopiramida y orciprenalina (117-119).

Es importante resaltar que, de acuerdo al consenso vigente, solo la quinidina y el isoproterol están recomendados para el manejo farmacológico del síndrome Brugada (120). Alogunos fármacos anestésicos logran mantener frecuencias cardíacas normales en
Tabla 1. Recomendaciones para el uso del DAI durante el tratamiento de un paciente con síndrome de Brugada según la Declaración del consenso sobre el diagnóstico y el tratamiento de pacientes con síndromes de arritmias primarias hereditarias

\section{Clase I}

Los siguientes cambios de estilo de vida se recomiendan en todos los pacientes con diagnóstico de $\mathrm{SBr}$.

Evitar fármacos que pueden inducir o agravar la elevación del segmento ST en derivaciones precordiales derechas.

Evitar la ingesta excesiva de alcohol.

Tratar la fiebre inmediatamente con medicamentos antipiréticos.

El DAI se recomienda en pacientes con diagnóstico de $\mathrm{SBr}$ que:

Son sobrevivientes de un paro cardíaco y/o,

Tienen una TV sostenida espontánea documentada con o sin síncope.

\section{Clase Ila}

El implante de DAI puede ser útil en pacientes con un ECG tipo I de diagnóstico espontáneo que tienen un historial de síncope, probablemente causado por arritmias ventriculares.

La quinidina puede ser útil en pacientes con un diagnóstico de $\mathrm{SBr}$ e historia de tormentas arrítmicas definidas como más de dos episodios de VT/V en 24 horas.

La quinidina puede ser útil en pacientes con un diagnóstico de SBr cuando:

El paciente califica para un DAI pero presenta una contraindicación para usarlo o se rehusa y/o,

El paciente tiene un historial de arritmias supraventriculares documentadas que requieren tratamiento.

La infusión de isoproterenol puede ser útil para suprimir las tormentas arrítmicas en pacientes con SBr.

\section{Clase IIb}

La implantación de DAI se puede considerar en pacientes con un diagnóstico de SBr que desarrollan FV durante la estimulación eléctrica programada.

La quinidina se puede considerar en pacientes asintomáticos con un diagnóstico de SBr con un ECG de tipo I espontáneo.

La ablación con catéter se puede considerar en pacientes con un diagnóstico de SBr e historia de tormentas arrítmicas o descargas repetidas del DAI.

\section{Clase III}

El implante de DAI no está indicado en pacientes con $\mathrm{SBr}$ asintomático con un ECG de tipo I inducido por fármacos, con historial familiar de solo una muerte cardiaca súbita.

Fuente: adaptado de Priori et al. (2013) 
los pacientes con $\mathrm{SBr}$ por sus propiedades sobre el sistema nervioso autónomo, su administración está encaminada a evitar la disfunción del canal de sodio cardíaco y a impedir algunas complicaciones como las arritmias (121). Sin embargo, el tratamiento farmacológico para el $\mathrm{SBr}$ no es muy efectivo (4). La implantación de un DAI previene la muerte súbita en pacientes sintomáticos con ECG tipo I $(122,123)$, además, si se presentan efectos desfavorables como eventos arrítmicos malignos tipo TV o FV, el DAI termina las anteriores situaciones con estimulación anti-taquicardia o con terapia de desfibrilación, de esta forma evita una parada cardiaca y posterior muerte súbita arrítmica (124).

Para los pacientes con el síndrome de Brugada que han sobrevivido a un paro cardíaco repentino o aquellos con un historial de síncope, que se cree es debido a taquiarritmias ventriculares, se recomienda la implantación de un DAI en lugar de un tratamiento farmacológico antiarrítmico, tal como se muestra en Ia (Figura 3).

\section{CONCLUSIONES}

En el continente americano no se han realizado estudios epidemiológicos sobre el $\mathrm{SBr}$, por lo que se desconoce la cifra exacta de presentación de casos. Se conoce que este síndrome es genéticamente heterogéneo, puesto que las causas genéticas son múltiples al presentarse mutaciones en diversos genes que codifican para los canales iónicos de sodio, potasio y calcio. Por esta razón la utilidad de las pruebas diagnósticas basadas en un solo marcador genético podría ser ambigua.

Se debe tener en cuenta que múltiples factores como el género, la edad, la historia familiar, el tipo y la penetrancia de la mutación pueden influir en el desarrollo del SBr y determinar su sintomatología.

Se han descrito algunos posibles inductores de patrones electrocardiográficos del $\mathrm{SBr}$, por ejemplo, episodios febriles causados por infecciones, ingesta de cocaína o fármacos que bloquean los canales de sodio, los cuales a su vez permiten desenmascarar la enfermedad.

La forma oculta o intermitente del síndrome dificulta el diagnóstico oportuno e incrementa el riesgo de sufrir muerte súbita (17). Se sabe que en reposo o durante el sueño puede ocurrir una disminución en la actividad simpática, lo que lleva a un aumento del tono parasimpático incrementando la posibilidad de sufrir una arritmia que podría conducir a la muerte del paciente.

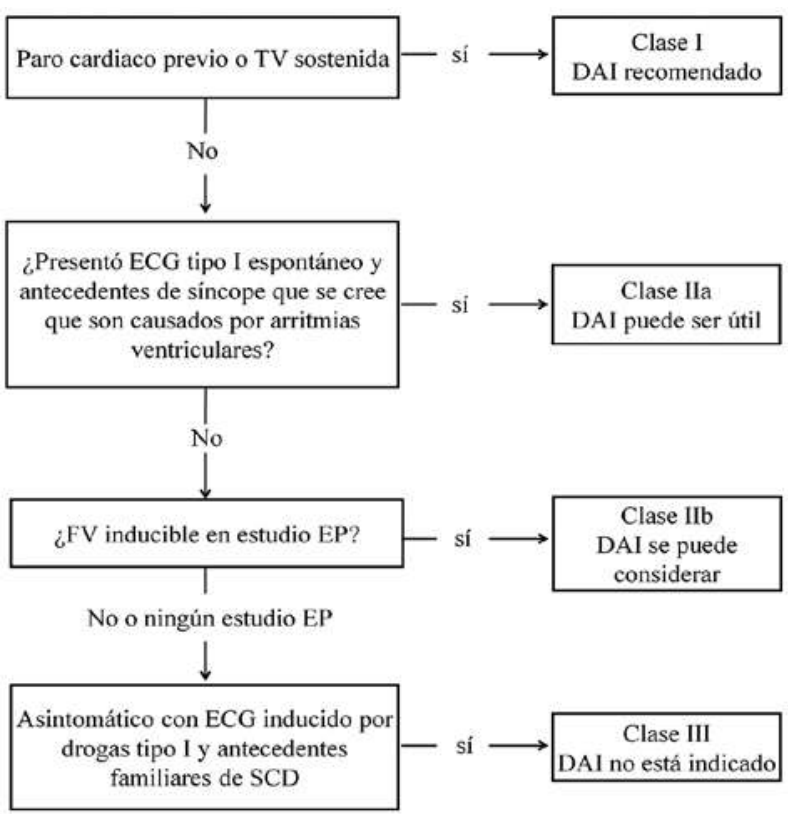

Figura 3. Algoritmo de manejo del DAl y uso de fármacos según la Declaración del consenso sobre el diagnóstico y el tratamiento de pacientes con síndromes de arritmias primarias hereditarias

ECG: electrocardiograma; EP: electrofisiología; DAl: desfibrilador automático inducible; SCD: muerte súbita cardiaca; FV: fibrilación ventricular; TV: taquicardia ventricular.Fuente: adaptado de Priori et al. (2013).

\section{PERSPECTIVASY RECOMENDACIONES}

Pese a los esfuerzos de investigación realizados en Colombia los reportes de pacientes con la enfermedad son escasos; es posible que una de las razones de los limitados registros esté relacionada con el reconocimiento de las manifestaciones clínicas del SBr, por lo que el síndrome pasa desapercibido en la mayoría de los casos. Por lo tanto, sería importante instruir al personal médico por parte de especialistas, con el fin de 
realizar un mejor diagnóstico, brindar el tratamiento adecuado a los pacientes y evitar mayor riesgo de muerte súbita por $\mathrm{SBr}$.

En Colombia no existen reportes sobre la expresión de este síndrome durante el embarazo, por lo que se sugiere la realización de estudios durante esta etapa, de esta manera se lograrían reconocer los síntomas que se presentan en este periodo y sería más comprensible el papel de las hormonas en el síndrome.

Es pertinente complementar estos estudios con investigaciones in vivo usando biomodelos, que permitan no solo comprender mejor los mecanismos por los cuales actúa el síndrome, sino también proponer tratamientos más efectivos para los pacientes.

\section{CONFLICTOS DE INTERESES}

Ninguno por declarar.

\section{REFERENCIAS BIBLIOGRÁFICAS}

1. Asenjo GR, Madariaga RR, Morris CR, Montagna MR, Núñez FJ, Ortiz OM, et al. Muerte súbita por fibrilación ventricular recuperada: ¿síndrome de Brugada?: Caso clínico. Rev Méd Chile. 1998 Jul;126(7):81421. DOI 10.4067/S0034-98871998000700009.

2. Benito B, Brugada J, Brugada R, Brugada P. Síndrome de Brugada. Rev Esp Cardiol. 2009;62(11):1297-315. DOI 10.1016/S0300-8932(09)73082-9.

3. Rodriquez RL, Gómez VA, Cruz FC, Sotelo OG, VaIverde EA. Síndrome de Brugada: reporte del primer caso diagnosticado en Costa Rica. Rev Costarric Cardiol. 2002 Abr;4(1):46-50.

4. Santos LF, Correia E, Rodrigues B, Nunes L, Costa A, Carvalho JL, et al. Spontaneous fluctuations between diagnostic and nondiagnostic ECGs in Brugada syndrome screening: Portuguese family with Brugada syndrome. Ann Noninvasive Electrocardiol. 2010 Oct;15(4):337-43. DOI 10.1111/j.1542474X.2010.00389.X.

5. García-Castro M, García C, Reguero JR, Miar A, Rubín JM, Álvarez $\nabla$, et al. Espectro mutacional del gen SCN5A en pacientes españoles con síndrome de Brugada. Rev Esp Cardiol. 2010;63(7):856-9. DOI 10.1016/ S0300-8932(10)70189-5.
6. Watanabe H, Minamino T. Genetics of Brugada syndrome. J Hum Genet. 2016 Jan;61(1):57-60. DOI 10.1038/jhg. 2015.97.

7. Del Val Martín D, Rodríguez D, Zamorano JL. Bradiarritmias: disfunción sinusal, bloqueo auriculoventricular y trastornos de la conducción intraventricular. Medicine. 2017 Sep;12(38):2267-74. DOI 10.1016/j. med.2017.07.002.

8. Urrea JK, Parra PF, Casanova ME. Reporte de un caso de fibrilación auricular como manifestación inicial de síndrome de Brugada. Rev Colomb Cardiol. 2016;23(4):301.

9. Dorticós F, Dorantes M, Arbaiza JL, Castro J, Zayas R, Quiñones M, et al. Síndrome de Brugada: experiencia cubana 2001. Arch Cardiol Mex. 2002 JulSep;27(3)72:203-8.

10. Matsuo K, Akahoshi M, Nakashima E, Suyama A, Seto $S$, Hayano $M$, et al. The prevalence, incidence and prognostic value of the Brugada-type electrocardiogram: a population-based study of four decades. J Am Coll Cardiol. 2001 Sep;38(3):765-70.

11. Paparella G, Sarkozy A, Brugada P. Brugada syndrome: the prognostic dilemma and value of sincope. Minerva Med. 2009 Aug; 100(4):307-19.

12. Delise P, Allocca G, Marras E, Sitta N, Sciarra L. [rugada syndrome: diagnosis and risk stratification. G Ital Cardiol (Rome). 2010 Oct;11(10 Suppl 1):107S-113S. Italian.

13. Ruiz MI, Marín E, Nogüe S, Sanz-Gallén P, Berne P, Mont L, et al. Detección de un síndrome de Brugada en un reconocimiento médico laboral. Med Segur Trab. 2011;57(224):265-9. DOI 10.4321/S0465546X2011000300007.

14. Ikeda T, Takami M, Sugi K, Mizusawa Y, Sakurada H, Yoshino H. Noninvasive risk stratification of subjects with a Brugada-type electrocardiogram and no history of cardiac arrest. Ann Noninvasive Electrocardiol. 2005 Oct;10(4):396-403.

15. Naseef A, Behr ER, Batchvarov VN. Electrocardiographic methods for diagnosis and risk stratification in the Brugada syndrome. J Saudi Heart Assoc. 2015 Apr;27(2):96-108. DOI 10.1016/j.jsha.2014.06.004.

16. Antzelevitch C, Brugada P, Borogrefe M, Brugada J, Brugada R, Corrado D, et al. Brugada syndrome: report of the second consensus conference: endorsed by the Heart Rhythm Society and the European Heart 
Rhythm Association. Circulation. 2005 Feb;111(5):65970. Erratum in: Circulation. 2005 Jul;112(4):e74.

17. Guevara-Valdivia ME, de Micheli A, Iturralde P, Colín L, Márquez MF, González-Hermosillo JA. Alteraciones electrocardiográficas infrecuentes durante la prueba de tolerancia al ejercicio en un paciente con síndrome de Brugada. Arch Cardiol Méx. 2003 Jul-Sep;73(3):212-7.

18. Jaramillo CJ, Perdomo LF, Cardona E, Londoño GA. Brugada syndrome in a patient with syncope. Rev Colomb Cardiol. 2010;17(4):177-86.

19. Nademanee K, Veerakul G, Nimmannit S, Chaowakul V, Bhuripanyo K, Likittanasombat K, et al. Arrhythmogenic marker for the sudden unexplained death syndrome in Thai men. Circulation. 1997 Oct;96(8):2595-600.

20. Dendramis G, Petrina SM, Baranchuk A. Not all STsegment elevations are myocardial infarction: Hyperkalemia and Brugada phenocopy. Am J Emerg Med. 2017 Apr;35(4):662.e1-662.e2. DOI 10.1016/j. ajem.2016.10.063.

21. Picón Heras R. Síndrome de Brugada: puesta al día. Cardiocore. 2013;48(2):69-74.

22. Silva D, Martins FM, Cavaco D, Adragão P, Silva MM, Anjos R, et al. Natural history of Brugada syndrome in a patient with congenital heart disease. Rev Port Cardiol. 2015 Jul-Aug;34(7-8):493.e1-4. DOI 10.1016/j. repc.2014.12.007.

23. Carrizo AG, Goransky A, Baranchuk A. Brugada phenocopy during right coronary artery dissection. J Electrocardiol. 2017 Nov-Dec;50(6):969-971. DOI 10.1016/j.jelectrocard.2017.07.005.

24. Chiale PA, Franco DA, Selva H, Militello CA. Supradesnivel persistente del segmento ST en las derivaciones precordiales derechas y muerte súbita por fibrilación ventricular en un paciente sin cardiopatía estructural (síndrome de Brugada). Revista Aroentina de Cardiologia 1999;67(5):645:8.

25. Genaro NR, Anselm DD, Cervino N, Estevez AO, Perona C, Villamil AM, et al. Brugada phenocopy clinical reproducibility demonstrated by recurrent hypokalemia. Ann Noninvasive Electrocardiol. 2014 JuI;19(4):387-90. DOI 10.1111/anec.12101.

26. Leiria TL, Mantovani A, de March Ronsoni R, Martins Pires L, Lapa Kruse M, Glotz de Lima G. Brugada syndrome after using cold medicine: is there any relation? Rev Port Cardiol. 2013 May;32(5):415-7. DOI 10.1016/j.repc.2012.11.005.
27. Ferrando-Castagnetto F, Garibaldi-Remuñan A, Vignolo G, Ricca-Mallada R, Baranchuk A. Brugada Phenocopy as a Dynamic Electrocardiographic Pattern during Acute Anterior Myocardial Infarction. Ann Noninvasive Electrocardiol. 2016 Jul;21(4):4258. DOI 10.1111/anec. 12351.

28. Echeverri D, Ramírez JD. Pacientes con episodios sincopales y síndrome de Brugada. Rev Iberoam Arritm. 2014;5(1):176-84.

29. Contreras E, Díaz JC, Zuluaga S. Síndrome de Brugada: descripción de un caso clínico. Rev Col Cardiol. 2007;14(2):113-116.

30. Argenziano M, Tiscornia G, Moretta R, Amorena CE, García Gras E. Control hormonal de las corrientes de la fase 1 del potencial de acción cardíaco en el síndrome de Brugada. Rev Argent Cardiol. 2014;82(4):310-5. DOI 10.7775/rac.es. $\nabla 82.14 .3885$.

31. Benito B, Berruezo A. Síndrome de Brugada y embarazo: indagando en el papel de las hormonas sexuales en las canalopatías iónicas. Rev Esp Cardiol. 2014;67(3):165-7. DOI 10.1016/j.recesp.2013.09.023.

32. Romero García AR, Castro Hevia J, Domínguez-Peréz RJ, Santos Hernádez AM, Ricardo Infanzón E, Arias Salazar W. Síndrome de Brugada en una mujer con presíncopes recurrentes. Reporte de caso. Rev Cubana Cardiol Cir Cardiovasc. 2014;20(3):158-65.

33. Rodríguez-Mañero M, Casado-Arroyo R, Sarkozy A, Leysen E, Sieira JA, Namdar M, et aI. Trascendencia clínica del embarazo en el síndrome de Brugada. Rev Esp Cardiol. 2014;67(3):176-80. DOI 10.1016/j.recesp.2013.06.026.

34. Crotti L, Marcou CA, Tester DJ, Castelletti S, Giudicessi JR, Torchio M, et al. Spectrum and prevalence of mutations involving BrS1- through BrS12-susceptibility genes in a cohort of unrelated patients referred for Brugada syndrome genetic testing: implications for genetic testing. J Am Coll Cardiol. 2012 Oct;60(15):1410-8. DOI 10.1016/j.jacc.2012.04.037.

35. Castro Hevia JA. Sexo femenino y Síndrome de Brugada. ¿Menor riesgo que los hombres? Rev Cuba Cardiol Cir Cardiovasc. 2014;19(3-4):1-4.

36. Shimizu W, Matsuo K, Kokubo Y, Satomi K, Kurita T, Noda T, et al. Sex hormone and gender difference-role of testosterone on male predominance in Brugada syndrome. J Cardiovasc Electrophysiol. 2007 Apr; 18(4):415-21. 
37. Yamaki M, Sato N, Okada M, Fujita S, Go K, Sakamoto $\mathrm{N}$, et al. A case of Brugada syndrome in which diurnal ECG changes were associated with circadian rhythms of sex hormones. Int Heart J. 2009 Sep;50(5):669-76.

38. Eckardt L. Gender differences in Brugada syndrome. J Cardiovasc Electrophysiol. 2007 Apr;18(4):422-4.

39. Marcus FI. Is the phenotypic expression of the brugada syndrome by the electrocardiogram different in men than in women? J Cardiovasc Electrophysiol. 2008 Nov;19(11):1186-7. DOI 10.1111/j.15408167.2008.01248.x.

40. Benito B, Sarkozy A, Mont L, Henkens S, Berruezo A, Tamborero D, et al. Gender differences in clinical manifestations of Brugada syndrome. J Am Coll Cardiol. 2008 Nov 4;52(19):1567-73. DOI 10.1016/j. jacc.2008.07.052.

41. Sieira J, Conte G, Ciconte G, de Asmundis C, Chierchia GB, Baltogiannis G, et al. Clinical characterisation and long-term prognosis of women with Brugada syndrome. Heart. 2016 Mar;102(6):452-8. DOI 10.1136/heartjnl-2015-308556.

42. Sacher F, Meregalli P, Veltmann C, Field ME, Solnon A, Bru P, et al. Are women with severely symptomatic brugada syndrome different from men? J Cardiovasc Electrophysiol. 2008 Nov;19(11):1181-5. DOI 10.1111/j.1540-8167.2008.01223.X.

43. Madeira M, Caetano F, Providência R, Almeida I, Trigo J, Nascimento J, et al. Fever-induced type 1 Brugada pattern. Rev Port Cardiol. 2015 Apr;34(4):287.e1-7. DOI 10.1016/j.repc.2014.10.002.

44. Tsarouhas K, Papalexis P, Kafantaris I, Tsitsimpikou Ch, Vavetsi S, Rentoukas E. Electrocardiographic findings compatible with Brugada syndrome in a patient with febrile respiratory infection. Hippokratia. $2010 \mathrm{Jul} ; 14(3): 221-3$.

45. Brito MR, Miranda CE, Rabelo W, Marino RL. Type 1 electrocardiographic Brugada pattern in a woman with Chagas disease: a case report. Europace. 2010 Sep;12(9):1345-6. DOI 10.1093/europace/euq129.

46. Arce M, Riera AR, Femenía F, Baranchuk A. Brugada electrocardiographic phenocopy in a patient with chronic Chagasic cardiomyopathy. Cardiol J. 2010;17(5):525-7.

47. Porres JM, Brugada J, Urbistondo V, García F, Reviejo $\mathrm{K}$, Marco P. Fever unmasking the Brugada syndrome. Pacing Clin Electrophysiol. 2002 Nov;25(11):1646-8.
48. Saura D, García-Alberola A, Carrillo P, Pascual D, Martínez-Sánchez J, Valdés M. Brugada-like electrocardiographic pattern induced by fever. Pacing Clin Electrophysiol. 2002 May;25(5):856-9.

49. Babuty D, Argibay J, Hatem S. Électrophysiologie cardiaque. Cardiologie. 2008;3(3):1-18. DOI 10.1016/ S1166-4568(08)47035-3

50. Arthur Guyton, Hall JE. Tratado de Fisiología Médica. $12^{\text {th }}$ ed. España: Elsevier; 2011.

51. Ganong W, Barrett K, Barman S, Boitano S. Ganong Fisiología Medica. 23 $3^{\text {th }}$ ed. Nueva York: McGraw-Hill; 2010.

52. Angarita BEV, Peña CLS, Neira MC. Principios de electrofisiología, arritmias. Colegio Colombiano de Electrofisiologia Cardiovascular. 2016;2:790-805.

53. Asensio E, Álvarez B, Lozano E, Farías A, Brugada R, Brugada P, et al. Elevación deI ST, Bloqueo de rama derecha 8 muerte súbita: Síndrome de Brugada. Arch Inst Cardiol Méx. 2000 May-Jun;70:301-11.

54. Campuzano Ó, Sarquella-Brugada G, Brugada R, Brugada P, Brugada J. Bases genéticas de las arritmias malignas y las miocardiopatías. Rev Esp Cardiol. 2009;62(4):422-36. DOI 10.1016/S0300-8932(09)70899-1.

55. Pérez-Riera A, Fortunato de Cano S, Fleury de Padua N, Schapachnik E. Síndrome de Brugada: nuevos conceptos y expectativas futuras. Rev Argent Cardiol. 2001;69:652-62.

56. Giménez NI, Foglia YC, Giménez NL, Aguirre A. Síndrome de Brugada: Revisión. Rev Posgrad. 2006;154:16-20.

57. Carmona Puerta R. Síndrome de Brugada: desde los genes hasta la terapéutica. CorSalud. 2010;2(2):145-61.

58. Nacur Lorentz M, Brandao Vianna BS. Arritmias Cardíacas y Anestesia. Rev Bras Anestesiol 2011;61(6):440-8.

59. Berne P, Aguinaga LE, Brugada J. Síndrome de Brugada. Rev Fed Arg Cardiol. 2012;41(4):225-34.

60. Gaztañagaa L, Marchlinskia FE, Betenskya BP. Mecanismos de las arritmias cardiacas. Rev Esp Cardiol. 2012;65(2):174-85. DOI 10.1016/j.recesp.2011.09.018.

61. Herbert E, Chahine M. Clinical aspects and physiopathology of Brugada syndrome: review of current concepts. Can J Physiol Pharmacol. 2006 AugSep;84(8-9):795-802.

62. de-Riva-Silva M, Montero-Cabezas JM, Fontenla-Cerezuela A, Salguero-Bodes R, López-Gil M, 
Arribas-Ynsaurriaga F. Respuesta positiva diferida al test de flecainida en paciente con sospecha de síndrome de Brugada: un hallazo preocupante. Rev Esp Cardiol. 2014;67(8):674-5. DOI 10.1016/j.recesp.2014.03.007.

63. Gasparini M, Priori SG, Mantica M, Napolitano C, Galimberti P, Ceriotti C, et al. Flecainide test in Brugada syndrome: a reproducible but risky tool. Pacing Clin Electrophysiol. 2003 Jan;26(1 Pt 2):338-41.

64. Meregalli PG, Ruijter JM, Hofman N, Bezzina CR, Wilde AA, Tan HL. Diagnostic value of flecainide testing in unmasking SCN5A-related Brugada syndrome. J Cardiovasc Electrophysiol. 2006 Aug; 17(8):857-64.

65. Díaz-Padrón Camejo R, Castro Hevia J, Quiñones Pérez M. Síndrome de Brugada. Rev Cubana Cardiol Cir Cardiovasc. 2000;14(2):133-40.

66. Hermosillo A, Cárdenas M, Márquez MF, Vallejo M. EI síndrome de Brugada y su relación con el sistema nervioso autónomo y el síncope vasovagal. Arch Cardiol Méx. 2008 Ene-Mar;78(1):7-10.

67. Nomura M, Nada T, Endo J, Kondo Y, Yukinaka M, Saito K, et al. Brugada syndrome associated with an autonomic disorder. Heart. 1998 Aug;80(2):194-6.

68. Juang JJ, Horie M. Genetics of Brugada syndrome. J Arrhythm. 2016 Oct;32(5):418-425.

69. Barajas-Martínez H, Hu D, Antzelevitch C. Bases genéticas y moleculares del síndrome de Brugada mediado por canales de sodio. Arch Cardiol Mex. 2013 OctDec;83(4):295-302. DOI 10.1016/j.acmx.2013.10.001.

70. Remme CA. Cardiac sodium channelopathy associated with SCN5A mutations: electrophysiological, molecular and genetic aspects. J Physiol. 2013 Sep;591(17):4099-116. DOI 10.1113/ jphysiol.2013.256461.

71. Abriel H. Cardiac sodium channel $\mathrm{Na}(\nabla) 1.5$ and interacting proteins: Physiology and pathophysiology. J Mol Cell Cardiol. 2010 Jan;48(1):2-11. DOI 10.1016/j. yjmcc.2009.08.025.

72. Nielsen MW, Holst AG, Olesen SP, Olesen MS. The genetic component of Brugada syndrome. Front Physiol. 2013 JuI;4:179. DOI 10.3389/fphys.2013.00179.

73. Hu D, Barajas-Martínez H, Pfeiffer R, Dezi F, Pfeiffer $\mathrm{J}$, Buch T, et al. Mutations in SCN10A are responsible for a large fraction of cases of Brugada syndrome. J Am Coll Cardiol. 2014 Jul;64(1):66-79. DOI 10.1016/j. jacc.2014.04.032.
74. Sieira J, Dendramis G, Brugada P. Pathogenesis and management of Brugada syndrome. Nat Rev Cardiol. 2016 Dec;13(12):744-56. DOI 10.1038/nrcardio.2016.143.

75. London B, Michalec M, Mehdi H, Zhu X, Kerchner L, Sanyal S, et al. Mutation in glycerol-3-phosphate dehydrogenase 1 like gene (GPD1-L) decreases cardiac $\mathrm{Na}+$ current and causes inherited arrhythmias. Circulation. 2007 Nov;1 16(20):2260-8.

76. Kattygnarath D, Maugenre S, Neyroud N, Balse E, Ichai C, Denjoy I, et al. MOG1: a new susceptibility gene for Brugada syndrome. Circ Cardiovasc Genet. 2011 Jun;4(3):261-8. DOI 10.1161/CIRCGENETICS.110.959130.

77. Ackerman MJ, Marcou CA, Tester DJ. Medicina personalizada: diagnóstico genético de cardiopatías/canalopatías hereditarias. Rev Esp Cardiol. 2013;66(4):298307. DOI 10.1016/j.recesp.2012.12.010.

78. Berne P, Brugada J. Brugada syndrome 2012. Circ J. 2012;76(7):1563-71.

79. IturraIde-Torres $\mathrm{P}$, Medeiros-Domingo A. Genética en los Síndromes de QT prolongado. Arch Cardiol Mex. 2009;79(Supl 2):26-30.

80. Cordeiro JM, Marieb M, Pfeiffer R, Calloe K, Burashnikov E, Antzelevitch C. Accelerated inactivation of the L-type calcium current due to a mutation in CACNB2b underlies Brugada syndrome. J Mol Cell Cardiol. 2009 May;46(5):695-703.

81. Monteforte N, Napolitanoa C, Priori SG. Genética y arritmias: aplicaciones diagnósticas y pronosticas. Rev Esp Cardiol. 2012;65(3):278-86. DOI 10.1016/j. recesp.2011.10.008

82. Arbelo E, Brugada J. Síndrome de Brugada 2013. Cuad Estim Cardiaca. 2013:3-13.

83. Schimpf R, Veltmann C, Wolpert C, Borogrefe M. Channelopathies: Brugada syndrome, long QT syndrome, short QT syndrome, and CPVT. Herz. 2009 Jun;34(4):281-8. DOI 10.1007/s00059-009-3238-1.

84. Abud A, Goyeneche R, Carlessi A, Strada B, Becker C. Posible valor pronóstico de la fibrilación y el flutter auricular en el síndrome de Brugada. Arch Cardiol Méx. 2013 Ene-Mar;83(1):4-7. DOI 10.1016/j. acmx.2013.01.012.

85. Vega J, Enríquez A, Vergara I, Frangini P, Baeza M, MiIlapán I, et al. [Electrical storm in Brugada syndrome successfully treated with isoproterenol. Report of a 
case]. Rev Med Chil. 2013 Oct;141(10):1340-3. DOI 10.4067/S0034-98872013001000016.

86. García Seara J, Martínez Sande JL, Rodríguez Mañero M, Fernández López XA, González Melchor L, González Juanatey RG. Tormenta arrítmica. Ritmo [internet]. 2017;2. [consultado 2019 mar 5]. Disponible en: https://bit.ly/2Tl2Sw3.

87. Brugada J, Brugada P, Brugada R. El síndrome de Brugada y las miocardiopatías derechas como causa de muerte súbita. Diferencias y similitudes. Rev Esp Cardiol. 2000;53(2):275-85.

88. Boda S, Mishra PC, Naik BS. Diagnosis of Brugada syndrome during fever: a case report from a tertiary care centre. J Assoc Physicians India. 2014 Jul;62(7):616-9.

89. Zaidi AN. An unusual case of Brugada syndrome in a 10-year-old child with fevers. Congenit Heart Dis. 2010 Nov-Dec;5(6):594-8. DOI 10.1111/j.17470803.2010.00382.X.

90. Gonzalez MC, Sieira J, Pappaert G, de Asmundis C. Chierchia GB, La Meir M, et al. Implantable Cardioverter-Defibrillators in Children and Adolescents With Brugada Syndrome. J Am Coll Cardiol. 2018 Jan;71(2):148-157. DOI 10.1016/j.jacc.2017.10.082.

91. Márquez MF, Allende R, Cazares-Campos I, Cárdenas M. Utilidad de las derivaciones paraesternales altas en el diagnóstico del Síndrome de Brugada. Arch Cardiol Méx. 2009 Dic;79(Supl 2):40-3.

92. Pérez-Riera AR, Femenía F, Baranchuk Facc A. Valor del electrocardiograma en el diagnóstico y pronóstico del síndrome de Brugada. Rev Urug Cardiol. 2011 Sep;26(2):122-40.

93. Carrillo-Esper R, Espinoza de los Monteros-Estrada I, Espinoza de los Monteros-Estrada GK. Síndrome de Brugada tipo II. Rev Mex Anestesiol. 2014;37(1):44-7.

94. Contreras E, Díaz JC, Zuluaga SX. Síndrome de Brugada: descripción de un caso clínico. Rev Col Cardiol. 2007 Mar-Abr; 14(2):113-6.

95. Jaramillo H, Acosta M, Aguilera R, Acosta H. Paciente con síndrome de Brugada con alteración en el gen SCN5A. Comunicación de un caso y revisión de la bibliografía. Med Int Mex. 2008 May-Jun;24(3):243-6.

96. Abrego HA, Suárez Arana R, Sandoval ÁE. Prevalencia de patrones electrocardiográficos tipo Brugada en un centro de monitoreo cardíaco transtelefónico en El Salvador. Rev Costarric Cardiol. 2006 May;8(2):13-7.
97. Guevara-Valdivia ME, Iturralde Torres P, de Micheli A, Huarte Hernández Y, Galvan L, Colín Lizalde L, et al. Desenmascaramiento del 'síndrome de Brugada' con propafenona endovenosa. Arch Cardiol Méx. 2002 Ene-Mar;72(1):45.

98. Castro Hevia JA. Actualización sobre el manejo de los pacientes con el patrón electrocardiográfico del síndrome de Brugada. Rev Cubana Invest Bioméd. 2012 Abr-Jun;31(2):175-86.

99. Keller DI, Rougier JS, Kucera JP, Benammar N, Fressart $\nabla$, Guicheney P, et al. Brugada syndrome and fever: genetic and molecular characterization of patients carrying SCN5A mutations. Cardiovasc Res. 2005 Aug;67(3):510-

100.López-Caleya JF, Fernández-Diéguez MO, MartínRodrigo L, Tuya-Morán MJ. Brugada tipo 1 asociado a celulitis: revisión y puesta al día. Semergen. 2016;42(3):e22-e4. DOI 10.1016/j.semerg.2015.01.016.

101. Daimi H, Khelil AH, Ben Hamda K, Aranega A, Chibani JB, Franco D. Absence of family history and phenotype-genotype correlation in pediatric Brugada syndrome: more burden to bear in clinical and genetic diagnosis. Pediatr Cardiol. 2015 Jun;36(5):1090-6. DOI 10.1007/s00246-015-1133-5.

102. Kasper D, Fauci A, Hauser S, Longo D, Jameson JL, Loscalzo J. Harrison: Principios de Medicina Interna. $19^{\text {th }}$ ed. España: McGraw-Hill; 2016.

103. García García G, González García S, Magro Ledesma D, Aranda López C. Patrón de Brugada tipo II desencadenado por fiebre secundaria a una pielonefritis aguda por Enterobacter aerogenes. Galicia Clin. 2013;74(1):23-4.

104. Adler A, Topaz G, Heller K, Zeltser D, Ohayon T, Rozovski U, et al. Fever-induced Brugada pattern: how common is it and what does it mean? Heart Rhythm. 2013 Sep;10(9):1375-82. DOI 10.1016/j.hrthm.2013.07.030.

105. Baranchuk A, Simpson CS. Brugada syndrome coinciding with fever and pandemic (H1N1) influenza. CMAJ. 2011 Mar;183(5):582. DOI 10.1503/ cmaj. 100016.

106. Serletis-Bizios A, Azevedo ER, Singh SM. Unmasking of Brugada syndrome during a febrile episode. Can J Cardiol. 2009 Apr;25(4):239.

107. Meggiolaro M, Zorzi A, El Maghawry M, Peruzza F, Migliore F, Pittoni GM. Brugada ECG disclosed by acute malaria: is it all about fever and propofol? J 
Clin Anesth. 2013 Sep;25(6):483-7. DOI 10.1016/j.jclinane.2013.02.012.

108.Suzuki T, Kohsaka S. Brugada-type electrocardiographic changes in a febrile patient of african descent. Am J Med Sci. 2006 Aug;332(2):97-9. DOI 10.1097/00000441-200608000-00010.

109. Patanè S, Marte F, La Rosa FC, Albanese A, La Rocca R, Villari SA. Revelation of Brugada electrocardiographic pattern during a febrile state. Int J Cardiol. 2010 Apr;140(1):e19-21. DOI 10.1016/j.ijcard.2008.11.069.

110.Monti M, Olivi G, Francavilla F, Borgognoni F. Pericarditis mimicking Brugada syndrome. Am J Emerg Med. 2017 Apr;35(4):669.e1-669.e3. DOI 10.1016/j. ajem.2016.11.015.

111. Mizusawa X, Morita H, Adler A, Havakuk O, Thollet A, Maury P, et al. Prognostic significance of feverinduced Brugada syndrome. Heart Rhythm. 2016 JuI;13(7):1515-20. DOI 10.1016/j.hrthm.2016.03.044.

112. Havakuk O, Viskin S. A Tale of 2 Diseases: The History of Long-QT Syndrome and Brugada Syndrome. J Am Coll Cardiol. 2016 Jan;67(1):100-8. DOI 10.1016/j. jacc.2015.10.020.

113. Postema PG, Wolpert C, Amin AS, Probst $\nabla$, Borggrefe $M$, Roden DM, et al. Drugs and Brugada syndrome patients: review of the literature, recommendations, and an up-to-date website (www.brugadadrugs.org). Heart Rhythm. 2009 Sep;6(9):1335-41. DOI 10.1016/j. hrthm.2009.07.002.

114. Pelegrín Martínez AÁ, Padró Suárez L. Marcapasos desfibrilador automático implantable en familiares con síndrome de Brugada. MEDISAN. 2011 Nor;15(1 1):1639-46.

115.Abud AM, Carlessi A, Goyeneche R, Strada B, Arceluz M, Fernández A, et al. Análisis retrospectivo de una población de pacientes con síndrome de Brugada y cardiodesfibrilador automático implantable. Rev Argent Cardiol. 2014 Feb;82(1):21-5.

116.Álvarez-Gómez JA, Sánchez MD, Stanley J, Beltranena RS, Hevia JC, Sarduy DB, et al. Marcadores eléctricos no invasores en pacientes con síndrome de Brugada. Arch Cardiol Mex. 2006 Ene-Mar;76(1):52-8.

117. Maury P, Hocini M, Haïssaguerre M. Electrical storms in Brugada syndrome: review of pharmacologic and ablative therapeutic options. Indian Pacing Electrophysiol J. 2005 Jan;5(1):25-34.

118. Schweizer PA, Becker R, Katus HA, Thomas D. Successful acute and long-term management of electrical storm in Brugada syndrome using orciprenaline and quinine/quinidine. Clin Res Cardiol. 2010 Jul;99(7):467-70. DOI 10.1007/s00392-010-0145-7.

119. González-Melchor L, Iturralde-Torres P, VillarrealMolina T, Medeiros-Domingo A. Muerte súbita cardiaca en el corazón estructuralmente normal: una actualización. Arch Cardiol Mex. 2014;84(4):293-304. DOI 10.1016/j.acmx.2014.04.002 1405-9940.

120. Priori SG, Wilde AA, Horie M, Cho Y, Behr ER, Berul C, et al. HRS/EHRA/APHRS expert consensus statement on the diagnosis and management of patients with inherited primary arrhythmia syndromes: document endorsed by HRS, EHRA, and APHRS in May 2013 and by ACCF, AHA, PACES, and AEPC in June 2013. Heart rhythm. 2013;10(12):1932-63. DOI 10.1016/j.hrthm.2013.05.014.

121.López-Jiménez FA, Mondragón-Villanueva ME. Síndrome de Brugada y anestesia. Rev Mex Anestesiol. 2008 Ene-Mar;31(1):55-62.

122. Mora G, Cuesta A, Mont L, García-Morán E, Francino A, Brugada J. Desfibrilador automático implantado en pacientes con miocardiopatía hipertrófica: criterios de selección, evolución y predictores de terapia apropiada. Rev Col Cardiol. 2007 May-Jul; 14(3):150-8.

123. Maron BJ, Shen WK, Link MS, Epstein AE, Almquist AK, Daubert JP, et al. Efficacy of implantable cardioverter-defibrillators for the prevention of sudden death in patients with hypertrophic cardiomyopathy. N Engl J Med. 2000 Feb;342(6):365-73.

124. Primo J, Geelen P, Brugada J, Filho AL, Mont L, WeIlens F, et al. Hypertrophic cardiomyopathy: role of the implantable cardioverter-defibrillator. J Am Coll Cardiol. 1998 Apr;31(5):1081-5. 